\title{
Microwave multiplex readout for superconducting sensors
}

\author{
E. Ferri ${ }^{\mathrm{a}, \mathrm{b}, *}$, D. Becker ${ }^{\mathrm{c}}$, D. Bennett ${ }^{\mathrm{c}}$, M. Faverzani ${ }^{\mathrm{a}, \mathrm{b}}$, J. Fowler $^{\mathrm{c}}$, J. Gard $^{\mathrm{c}}$, A. Giachero $^{\mathrm{a}, \mathrm{b}}$, J. Hays-Wehle $^{\mathrm{c}}$, G. Hilton $^{\mathrm{c}}$,

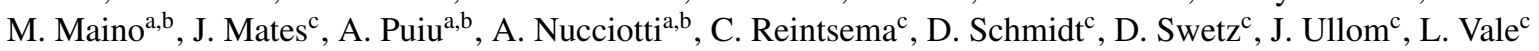 \\ ${ }^{a}$ Università Milano-Bicocca, Milan, Italy \\ ${ }^{b}$ INFN Sez. di Milano-Bicocca, Milan, Italy \\ ${ }^{c}$ NIST, Boulder, CO, USA
}

\begin{abstract}
The absolute neutrino mass scale is still an outstanding challenge in both particle physics and cosmology. The calorimetric measurement of the energy released in a nuclear beta decay is a powerful tool to determine the effective electron-neutrino mass. In the last years, the progress on low temperature detector technologies has allowed to design large scale experiments aiming at pushing down the sensitivity on the neutrino mass below $1 \mathrm{eV}$. Even with outstanding performances in both energy $(\sim \mathrm{eV}$ on $\mathrm{keV})$ and time resolution $(\sim 1 \mu \mathrm{s})$ on the single channel, a large number of detectors working in parallel is required to reach a sub-eV sensitivity. Microwave frequency domain readout is the best available technique to readout large array of low temperature detectors, such as Transition Edge Sensors (TESs) or Microwave Kinetic Inductance Detectors (MKIDs). In this way a multiplex factor of the order of thousands can be reached, limited only by the bandwidth of the available commercial fast digitizers. This microwave multiplexing system will be used to readout the HOLMES detectors, an array of 1000 microcalorimeters based on TES sensors in which the ${ }^{163}$ Ho will be implanted. HOLMES is a new experiment for measuring the electron neutrino mass by means of the electron capture (EC) decay of ${ }^{163} \mathrm{Ho}$. We present here the microwave frequency multiplex which will be used in the HOLMES experiment and the microwave frequency multiplex used to readout the MKID detectors developed in Milan as well.
\end{abstract}

Keywords: neutrino mass, microwave multiplexed readout, low temparature detectors

PACS: 14.60.Pq, 07.20.Mc, 84.40.Dc, 29.40.Vj

\section{Introduction}

In the last years new experiments aiming at pushing down the ${ }^{23}$ sensitivity on neutrino mass below $1 \mathrm{eV}$ have been designed. ${ }_{25}$ These experiments require a large number of detectors working ${ }_{26}$ in parallel, each characterized by marvelous time and energy resolution. The best available technique to readout large array of Transition Edge Sensors (TESs) or Microwave Kinetic Inductance Detectors (MKIDs) is the microwave frequency read- ${ }^{29}$ out. This technique will be used to readout the HOLMES [1] ${ }_{31}$ detectors, an array of 1000 microcalorimeters for measuring the neutrino mass by means of the electron capture (EC) decay of ${ }_{33}^{32}$ ${ }^{163}$ Ho. The HOLMES detectors will be Mo/Cu Transition Edge ${ }_{34}^{33}$ Sensor (TES) on $\mathrm{SiN}_{x}$ membrane with bismuth absorbers, in ${ }_{35}$ which the ${ }^{163}$ Ho nuclei will be fully embedded.

\section{HOLMES $\mu$ Mux readout and data acquisition}

A multiplexing system will be used for reading out the signals coming from the 1000 microcalorimeters of HOLMES. Even if the baseline for the HOLMES experiment is to use ${ }_{41}$ a Code Division Multiplexing (CDM) which has been devel- ${ }_{42}$ oped by NIST [2], recent advantages on microwave multiplex- ${ }_{43}$ ing ( $\mu$ Mux) suggest that this is the most suitable system for ${ }_{44}$

\footnotetext{
${ }^{*}$ Corresponding author

Email address: elena.ferri@mib.infn.it (E. Ferri )
} $\mathrm{n}$ ing factor. Microwave multiplexing [3, 4] is based on radio frequency (rf) SQUID and it allows independent optimization of the multiplexer and detectors. Furthermore, it does not degrade the sensitivity of the TES detectors. Each rf-SQUID is inductively coupled to a microwave superconducting resonator. Many RF tones are multiplexed by tuning each resonator to a unique frequency. The method for linearizing the response of the rf-SQUID is the flux-ramp modulation [5]. It consists in applying a periodic ramp that sweeps through multiple flux quanta in the SQUIDs. Therefore, an input signal looks like a flux offset during the duration of the ramp and produces a phase-shift in the rf-SQUID response.

The complete set up is composed by three different chips: the TES chip, the $\mu$ Mux board and the central interface (IF) board. On the multiplexer chip quarter-wave coplanar waveguide (CPW) microwave resonators are present as well as the rfSQUIDs, one for each detector. Each rf-SQUID acts as a fluxdependent nonlinear inductor. The IF board connects the TES to the input coil of the rf-SQUID present on the multiplexer board. This board also provides bias shunt resistors in parallel to each TES and a selectable inductance in series to increase the pulse rise times. A particle interaction in a constant voltage biased TES changes its resistance and as a consequence the current which flows in it. The TES current couples flux into the 


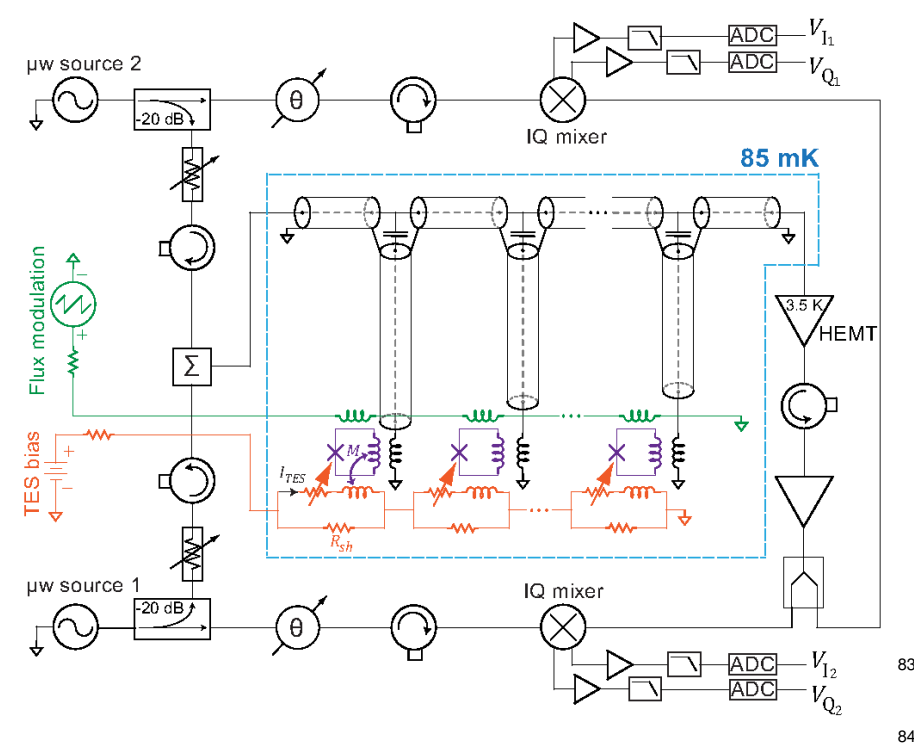

Figure 1: Circuit schematic for a two channel microwave multiplexed readout 85 of a TES array. Figure from [6].

rf-SQUID through an input coil with mutual inductance $M$. The ${ }_{89}$ rf-SQUID transduces a change in input flux into a change of ${ }_{90}$ phase of the periodic steady response of the rf-SQUID, obtained ${ }_{91}$ by demodulating the radio frequency signal with the homodyne ${ }_{92}$ technique. Up to now the TES microcalorimeter $\mu$ MUX princi- ${ }_{93}$ ple has been demonstrated in [6] with two channels.

The $\mu \mathrm{Mux}$ is suitable for a fully digital approach. The comb ${ }_{95}$ of frequencies tones is generated by digital synthesis in the 90 $\mathrm{MHz}$ range and up-converted to the GHz range by an IQ-mixer. 97 The comb is then sent to the $\mu$ Mux chip coupled to the TES ar- 98 ray, amplified by a low noise High Mobility Transistor (HEMT) 99 amplifier and sent back to room temperature. The output sig-100 nal is then down-converted by another IQ-mixer and sampled 101 with a fast A/D converter. To recover the signals of each TES 102 digital mixing techniques are used. To do so HOLMES will 103 use the Reconfigurable Open Architecture Computing Hard-104 ware $(\mathrm{ROACH} 2)$ board equipped with a FPGA Xilinx Virtex6 [7]. ROACH2, developed in the framework of CASPER (Collaboration for Astronomy Signal Processing and Electronic Re- ${ }^{105}$ search), is a fully open source project. The FPGA installed on ${ }_{106}$ the ROACH2 board will be programmed to perform a real-time ${ }_{107}$ digital signal processing. The complete acquisition system is ${ }_{108}$ composed by the ROACH2 board coupled to a DAC for the ${ }_{109}$ comb generation, ADC for signal digitization and an IF board ${ }_{110}$ for signal up and down conversion. The ADC boards currently ${ }_{111}$ available for ROACH2 are characterized by a sampling rate of $550 \mathrm{MS} / \mathrm{s}$ and a resolution of 12 bit.

The number of TES detectors which can be multiplexed on 114 one ROACH2 board is set by the bandwidth of both TES signa ${ }^{115}$ and the $\mathrm{A} / \mathrm{D}$ converter. Indeed, the multiplexing factor is ap ${ }^{116}$ proximately given by $0.02 f_{\text {adc }} \tau_{\text {rise }}$, where $f_{\text {adc }}$ is the sampling 118 frequency and $\tau_{\text {rise }}$ is the rise time (10-90\%) of a TES signal. A ${ }^{119}$ multiplexing factor of about 50 could be obtained with the per- ${ }^{120}$ formances of the ROACH 2 digitizer and a rise time of around 5 $\mu \mathrm{s}$.

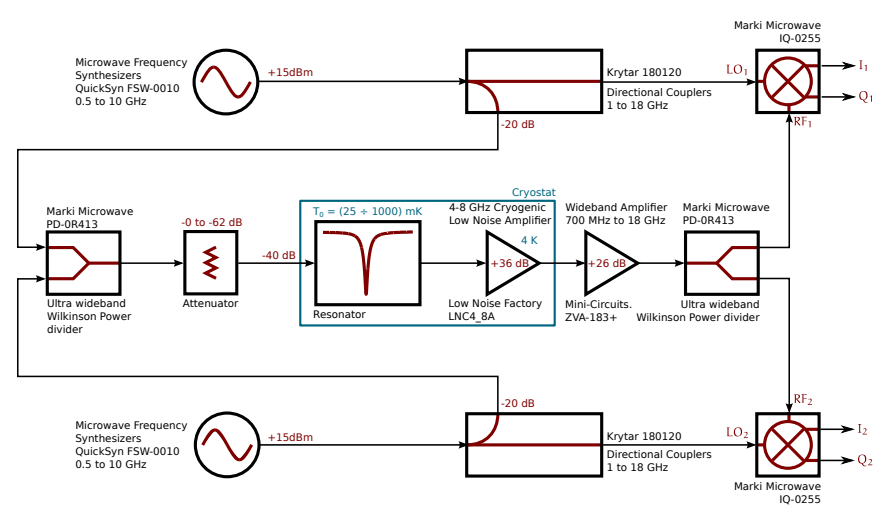

Figure 2: The 2-channel homodyne detection scheme employed for the readout.

\section{RF set up at Milano-Bicocca}

In Milan we have developed a RF circuit capable to readout two resonators at the same time (figure 2). The technique to read them out is based on the homodyne detection method, as reported in [8]. The microwave signals used to probe the resonators are generated by two synthesizers with a power of +15 $\mathrm{dBm}$. Each signal is split by means of a directional coupler: one copy is used as local oscillator (LO) of the IQ mixer used for the demodulation, while the other copy is attenuated by $20 \mathrm{~dB}$ and combined with the signal coming from the second synthesizer by a Wilkinson power divider. The obtained signal is transmitted inside the cryostat to the microresonator chip through a coax cable. The transmitted signal is then amplified by a factor $+36 \mathrm{~dB}$ by the HEMT amplifier and sent back at room temperature by another coax cable. Later, the signal is amplified by a room temperature amplifier (gain $+26 \mathrm{~dB}$ ), split in two by another power divider and sent to the RF ports of the IQ mixers. The mixers outputs I and Q are ready to be acquired and digitalized.

This RF set up has been used to acquire TES pulses with the microwave multiplexing technique. All the chips - i.e the TES chip, the $\mu$ Mux board and the IF board - are fabricated by NIST.

\section{Acknowledgments}

The HOLMES experiment is funded by the European Research Council under the European Union's Seventh Framework Programme (FP7/2007-2013)/ERC Grant Agreement no. 340321. We also acknowledge support from INFN for the MARE project, from the NIST Innovations in Measurement Science program for the TES detector development.

[1] B. Alpert et al, Eur. Phys. J C 75 (2015) 112.

[2] G.M. Stiehl et al, Applied Phys. Lett. 100 (2012) 072601.

[3] K. D. Irwin and K.W. Lehnert, Appl. Phys. Lett. 85 (2004) 2107-2109.

[4] J.A.B. Mates et al, Appl. Phys. Lett. 92 (2008) 023514.

[5] J.A.B. Mates, The Microwave SQUID Multiplexer, Ph.D. thesis, University of Colorado, Boulder (2011).

[6] O. Noroozian et al, Applied Phys. Lett. 103 (2013) 202602.

[7] S. McHugh et al, Rev. Sci. Instrum. 83 (2012) 044702.

[8] P.K. Day et al, Nature 425 (2003) 817-821. 